\title{
Imaging aspects of the racemose neurocysticercosis
}

\author{
Dequitier Carvalho Machado ${ }^{1,2}$, Gustavo Bittencourt Camilo ${ }^{1,2}$, Ursula David Alves ${ }^{2}$, \\ Celso Estevão de Oliveira ${ }^{2}$, Romulo Varella de Oliveira² ${ }^{2}$ Agnaldo José Lopes ${ }^{1}$
}

\author{
${ }^{1}$ Postgraduate Program in Medical Sciences, State University of Rio de Janeiro, \\ Rio de Janeiro, Brazil \\ 2Department of Radiology, State University of Rio de Janeiro, Rio de Janeiro, Brazil
}

Submitted: 4 February 2014

Accepted: 14 April 2014

Arch Med Sci 2015; 11, 6: 1356-1360

DOI: $10.5114 /$ aoms.2015.56366

Copryright $\odot 2015$ Termedia \& Banach

Neurocysticercosis (NCC) is the most common parasitic infection of the central nervous system (CNS) [1, 2]. It is estimated that approximately 50 million people are infected with NCC in developing countries, and this disease is considered endemic in Latin America, Asia and Africa [1]. Typically, this infection affects the brain parenchyma, manifesting as a focal lesion with adjacent edema. This condition rarely affects the ventricular system and subarachnoid space, although in cases where it does involve these regions, it is referred to as racemose NCC, which can rarely be observed by computed tomography (CT) [3]. Here, we report a case of racemose NCC in which neuroimaging findings were of critical importance for establishing the diagnosis of this rare form of NCC.

A 47-year-old man presented with dyslipidemia and systemic hypertension and had been monitored for nearly 32 years due to epilepsy that started during childhood. He reported a week-long evolution of a clinical condition consisting of frontal headache, with partial improvement following the use of anti-inflammatories, and his symptoms were associated with insomnia and amnesia and demonstrated progressive worsening. During the evolution of his clinical condition, he presented several recurrent focal dyscognitive seizures, eyelid and perioral myoclonia and motor aphasia lasting seconds; as a result, he was admitted to our hospital for investigation. Empirical treatment for neurotoxoplasmosis and seizure control was initiated with phenytoin, phenobarbital and valproic acid.

Laboratory tests were performed, and the results were nonspecific with a complete blood count showing leukocytosis with a predominance of lymphocytes. Serology results for HIV, human T-lymphotropic virus (HTLV), syphilis, toxoplasmosis, hepatitis A, B and C, and cytomegalovirus were negative, and the tuberculin skin test was nonreactive. An electroencephalogram showed an irregular alpha rhythm of 9 to $11 \mathrm{~Hz}$, at a low voltage, and also revealed two frontal epileptic seizures that started in the left hemisphere and spread to the homologous regions and bilateral temporal regions. During the epileptic seizures, the $\alpha$ rhythm was maintained, indicating that there was not complete loss of consciousness (only compromised consciousness).

High-resolution CT (HRCT) with the administration of iodinated contrast medium revealed small rounded lesions, which were well-defined, grouped, presented a 'grape-cluster' appearance and were located at the cortical-subcortical region of the left frontal lobe (superior and middle gyri). There was ring enhancement of the contrast medium associated with significant perilesional edema, as well as effacement of

\author{
Corresponding author: \\ Agnaldo José Lopes MD, PhD \\ Postgraduate Program \\ in Medical Sciences \\ State University \\ of Rio de Janeiro \\ Rua Araguaia, 1266 \\ Bloco $1 / 405$ \\ Freguesia, Jacarepaguá \\ 22745-271, Rio de Janeiro, \\ Brazil \\ Phone: +55212125762030 \\ E-mail: agnaldolopes.uerj@ \\ gmail.com
}


the adjacent cortical sulci and the corresponding lateral ventricle which caused contralateral deviation from the midline at approximately $0.8 \mathrm{~cm}$. One of the lesions showed gross calcifications (Figure 1). Imaging aspects were compatible with racemose NCC at the granular nodular and calcified nodular stages. At this time, treatment for NCC was initiated with albendazole and dexamethasone.

Two days after HRCT, magnetic resonance imaging (MRI) was performed to obtain T1-, T2-, $\mathrm{T}^{*}{ }^{*}$ and fluid-attenuated inversion recovery (FLAIR)-weighted sequences before and after the use of contrast medium. These images showed multinodular intra-axial lesions located at the left frontal lobe and the cortical-subcortical region, which were isointense and presented markedly hypointense halos on $\mathrm{T} 2$ and an intermediate signal and intense enhancement on T1. There was also severe perilesional edema with a fronto-parietal distribution (Figure 2). These imaging aspects were again consistent with racemose NCC.

Surgical brain biopsy of the left frontal region was performed, and two fragments with a hard consistency and light-brown color were collected. Microscopic analysis showed chronic inflammation with regions of focal necrosis and microabscesses. Although these findings were nonspecific, the clinical aspect was considered compatible with NCC. After histopathological analysis, treatment for neurotoxoplasmosis was interrupted, and treatment for NCC was maintained. The patient presented clinical improvement and was discharged 41 days after admission; he continued to receive valproic acid and achieved adequate control of his epileptic seizures.

Neurocysticercosis is a zoonosis caused by Taenia solium, for which humans serve as the only definitive host. Neurocysticercosis occurs when humans act as an intermediate host, acquiring parasite eggs through the ingestion of contaminated food. In this case, the larvae, in the form of cysticerci, are deposited in the nervous tissue $[2,4]$. The clinical manifestations are nonspecific and vary according to the location, size and number of cysts, as well as the immune response of the patient [2]. However, most patients with NCC present seizures.

The cysticerci may develop into two forms in the nervous system, including the cellulosae and racemose forms. The cellulosae form is the most common, where spherical vesicles of 1 to $2 \mathrm{~cm}$ may be observed at the cortical-subcortical interface. In this form, a discrete chronic inflammatory reaction occurs. In the racemose form, the cysticerci are located at the basal cisterns or the ventricular and subarachnoid spaces and usually present a multilobular vesicular form with a mass effect [4].

The development of modern neuroimaging techniques has dramatically altered the accuracy of NCC diagnosis, which may be associated with several neurological syndromes [5-7]. In racemose NCC, the lesions typically present a cystic aspect with a 'grape-cluster' appearance when reaching large sizes; these characteristics led to the term racemose NCC. This condition is associated with an intense inflammatory reaction, fibrosis and progressive thickening of the leptomeninges [8].
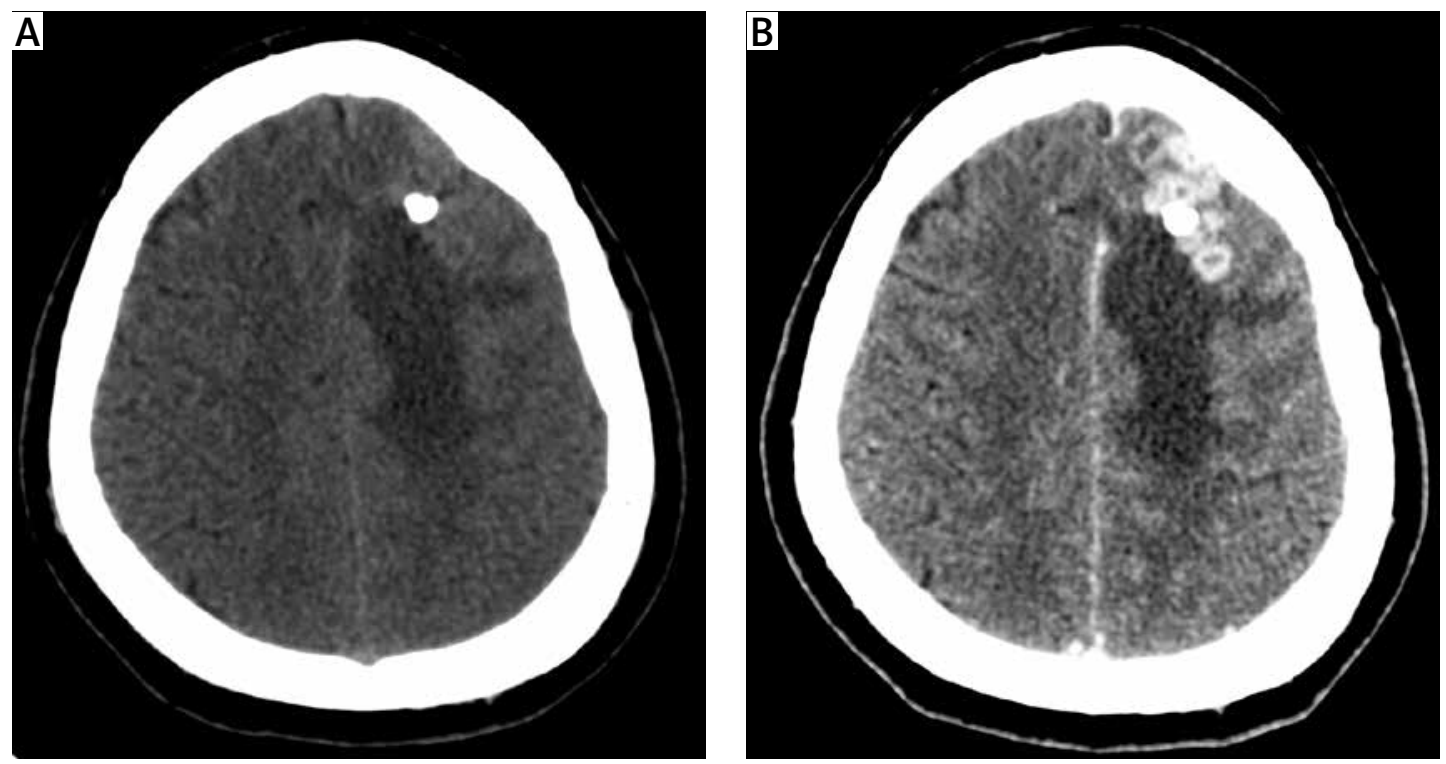

Figure 1. Cortical-subcortical left frontal lesion intermingled with gross calcification and edema/infiltration in the surrounding area, compressing the lateral ventricles and displacing the midline structures. A - Axial HRCT of the skull without contrast (W: 45; C: 90) demonstrating a left frontal lesion intermingled with gross calcification and edema/infiltration in the surrounding area. B - Axial HRCT of the skull after contrast (W: 45; C: 90) demonstrating multinodular lesions with intense enhancement of the ring type 

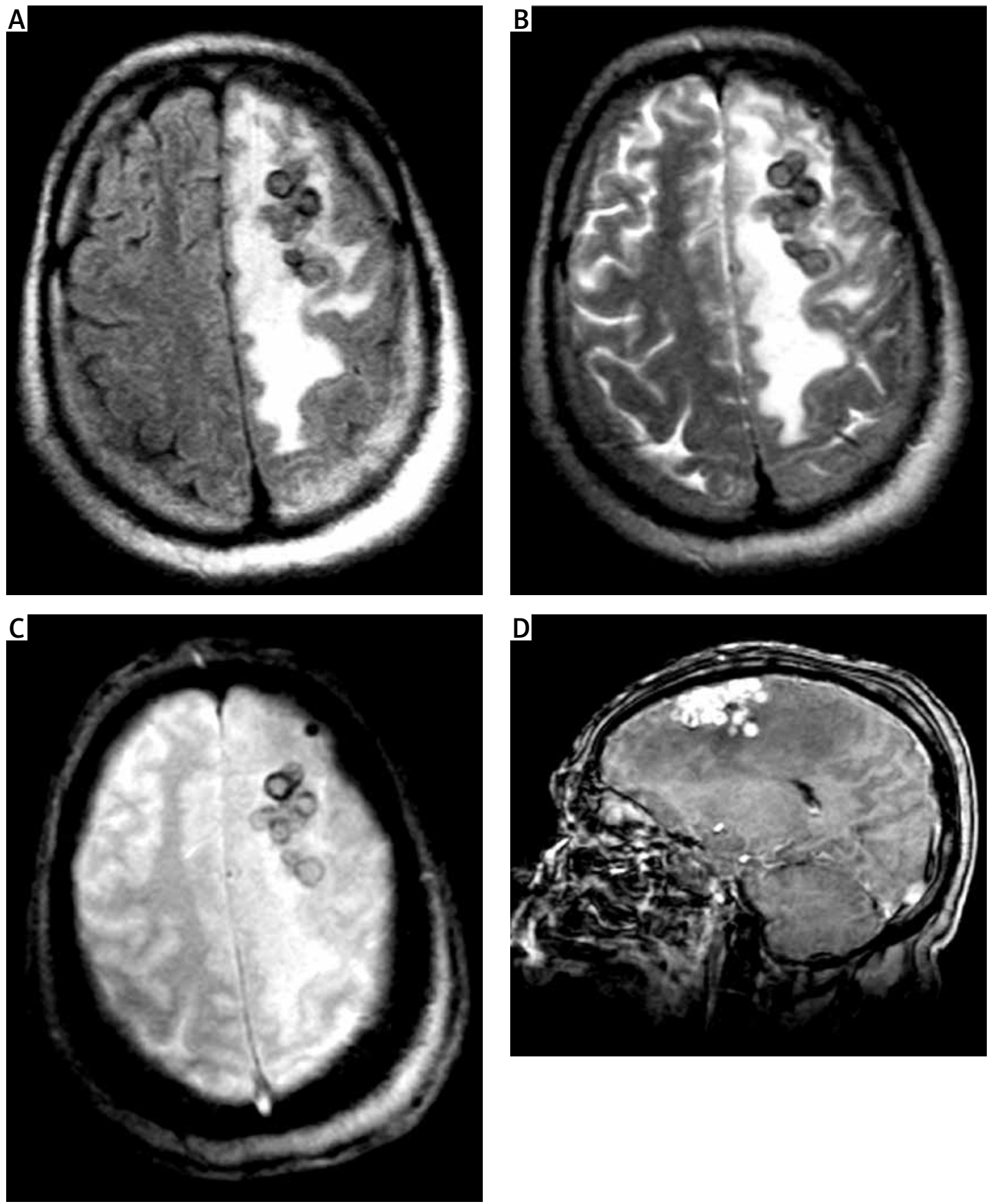

Figure 2. Magnetic resonance images of the skull with FLAIR- (A), T2*- (B), T2- (C) and T1- weighted sequences (D) following the intravenous administration of gadolinium. The sequence of images shows multinodular intra-axial lesions with a 'grape-cluster' appearance, which were isointense and demonstrated markedly hypointense halos on T2 and an intermediate signal with intense enhancement on T1. These lesions were located in the left frontal lobe and cortical-subcortical region. There was intense perilesional edema with a fronto-parietal distribution

In approximately $50-60 \%$ of cases, there is obstruction of the cerebrospinal fluid flow, resulting in hydrocephaly and progressive intracranial hypertension. Moreover, death occurs in over $20 \%$ of such cases, and this is therefore considered a malignant form of NCC $[9,10]$.

Computed tomography and MRI provide objective evidence concerning the number, topography and involution stage of the lesions [11]. Cystic lesions located within the cerebrospinal fluid cisterns usually have a multilobular appearance, tend to displace neighboring structures and behave as lesions with a mass effect [8]. Magnetic resonance imaging is the most valuable non-invasive method to demonstrate segmental narrowing or occlusion of intracranial arteries in patients with subarachnoid NCC [12]. However, cysts tend to demonstrate an intensity signal similar to that of 
the cerebrospinal fluid and usually do not show significant enhancement after the use of gadolinium. In CT, cysticerci appear as hypodense lesions that distort the ventricular system causing asymmetric hydrocephaly [13-15]. According to an evolutionary point of view, parenchymal NCC may be divided into the following four stages: (1) the vesicular stage, characterized by the presence of a scolex within the cyst and the absence of enhancement of the cyst wall; (2) the colloidal vesicular stage, characterized by thick fluid inside the cyst, a ring-enhancing lesion and loss of scolex; (3) the granular nodular stage, characterized by enhancement of the nodular lesion or a small ring-enhancing lesion; and (4) the nodular calcified stage, in which small, calcified granulomatous lesions can be seen [11].

There is a rather wide range of differential diagnoses of NCC. In the vesicular stage of this disease, the scolex is visible; its appearance is typical and practically pathognomonic. However, if the scolex is not detected, the following alternative features should be considered: widened perivascular spaces and neuroglial cysts in cases involving intraparenchymal lesions; ependymal cysts and choroid plexus cysts at intraventricular sites; and subarachnoid cysts and epidermoid cysts at other extra-axial locations $[16,17]$. In the colloidal vesicular stage of NCC, cystic metastases and primary neoplasms should be considered; these lesions are typically heterogeneous and have irregular margins, facilitating differential diagnosis in most cases [18]. Particular attention should be devoted to the possibility of pyogenic brain $a b$ scesses due to these abscesses' cortico-subcortical location, heterogeneous content and thin contrast-enhanced capsules. Among potential types of infections, fungal, parasitic and mycobacterial infections may present as low-density lesions with peripheral enhancement in $\mathrm{CT}[17,18]$. In addition to the aforementioned types of infections, subacute infarcts and parenchymal hematomas could exhibit ring-like enhanced lesions similar to NCC-related lesions in certain cases $[16,18,19]$. In the granular nodular stage of NCC, the main imaging characteristics for differential diagnosis include early stage granulomatous diseases and metastases that present as contrast-enhanced nodular lesions [16-20]. Finally, in the nodular calcified stage, late-stage granulomatous disease and tuberous sclerosis are the main conditions that should be considered in differential diagnoses of NCC [21-23].

In conclusion, the present case report describes a rare form of NCC. The lesions extended from the periventricular region to the cortical-subcortical interface, indicating a case of racemose NCC with an atypical location that was in the most chronic stage of the disease. Thus, we emphasize the need to explore neuroimaging methods to more comprehensively characterize the involvement of the CNS in this condition.

\section{Conflict of interest}

The authors declare no conflict of interest.

\section{References}

1. Guimarães RR, Orsini M, Guimarães RR, et al. Neurocysticercosis: an old disease update. Rev Neurocienc 2010; 18: 581-94.

2. Kim SW, Kim MK, Oh SM, Park SH. Racemose cyzticercosis in the cerebral hemisphere. J Korean Neurosurg Soc 2010; 48: 59-61.

3. Mittal P, Mittal G. Intraventricular and subarachnoid racemose cysticercosis. Trop Parasitol 2011; 1: 111-2.

4. Kumar V, Abbas AK, Fausto N. Robbins and Cotran: pathologic basis of disease. 8th ed. Rio de Janeiro: Elsevier 2007; 1480.

5. Carpio A, Escobar A, Hauser WA. Cysticercosis and epilepsy: a critical review. Epilepsia 1998; 39: 1025-40.

6. García HH, Del Brutto OH. Imaging findings in neurocysticercosis. Acta Tropica 2003; 87: 71-8.

7. García HH, Del Brutto OH, Nash TE, White AC Jr, Tsang VC, Gilman RH. New concepts in the diagnosis and management of neurocysticercosis (Taenia solium). Am J Trop Med Hyg 2005; 72: 3-9.

8. Fleury A, Carrillo-Mezo R, Flisser A, Sciutto E, Corona T. Subarachnoid basal neurocysticercosis: a focus on the most severe form of the disease. Expert Rev Anti Infect Ther 2011; 9: 123-33.

9. Pittella JEH. Neurocysticercosis. Brain Pathol 1997; 7: 681-93.

10. Estañol B, Corona T, Abad P. A prognostic classification of cerebral cysticercosis: therapeutic implications. J Neurol Neurosurg Psychiatry 1986; 49: 1131-4.

11. Lerner A, Shiroishi MS, Zee CS, Law M, Go JL. Imaging of neurocysticercosis. Neuroimaging Clin N Am 2012; 22: 659-76.

12. Del Brutto OH. Stroke and vasculitis in patients with cysticercosis. In: Uncommon causes of stroke. Caplan LR (ed.). Cambridge University Press, New York 2008; 53-8.

13. Lucato LT, Guedes MS, Sato JR, Bacheschi LA, Machado LR, Leite CC. The role of conventional MR imaging sequences in the evaluation of neurocysticercosis: impact on characterization of the scolex and lesion burden. AJNR Am J Neuroradiol 2007; 28: 1501-4.

14. Del Brutto OH. Neurocysticercosis: a review. Sci World J 2012; 2012: 159821.

15. Kimura-Hayama ET, Higuera JA, Corona-Cedillo R, et al. Neurocysticercosis: radiologic-pathologic correlation. RadioGraphics 2010; 30: 1705-19.

16. do Amaral LL, Ferreira RM, Rocha AJ, Ferreira NP. Neurocysticercosis: evaluation with advanced magnetic resonance techniques and atypical forms. Top Magn Reson Imaging 2005; 16: 127-44.

17. Osborn AG, Preece MT. Intracranial cysts: radiologicpathologic correlation and imaging approach. Radiology 2006; 239: 650-64.

18. Litt AW, Mohuchi T. Case 10: neurocisticercose. Radiology 1999; 211: 472-76.

19. Zeng J, Zheng P, Xu J, et al. Prediction of motor function by diffusion tensor tractography in patients with basal ganglion haemorrhage. Arch Med Sci 2011; 7: 310-4. 
20. Cheng J, Yu H, Wang L, Wang X, Shen G. Primary oral and maxillofacial liposarcoma: a clinicopathological and immunohistochemical study of eleven cases. Arch Med Sci 2012; 8: 316-23.

21. Kıroğlu Y, Callı C, Karabulut N, Oncel C. Intracranial calcifications on CT. Diagn Interv Radiol 2010; 16: 263-9.

22. Alkan A, Sharifov R, Guler S, Aralasmak A, Kocer A, Yuksel A. Involvement of the corpus callosum splenium in a case with SSPE: magnetic resonance spectroscopy findings. Arch Med Sci 2013; 9: 386-7.

23. Bruzgielewicz A, Rzepakowska A, Osuch-Wójcikewicz E, Niemczyk K, Chmielewski R. Tuberculosis of the head and neck - epidemiological and clinical presentation. Arch Med Sci 2014; 10: 1160-6. 